\title{
Gewerkschaften in Sri Lanka: Strukturen und Bündnispolitik unter der UNP-Regierung
}

\author{
Von Stefan Chrobot
}

\section{Ausgangslage und Uberblick}

Im Juli 1977 löste die United National Party (UNP) unter Führung von J. R. Jayewardene nach einem überwältigenden Wahlsieg die regierende Sri Lanka Freedom Party (SLFP) von Frau Bandaranaike ab. Als Koalitionspartner der SLFP waren bereits im September 1975 die Lanka Samasamaja Party (LSSP) und im Februar 1977 die Communist Party of Sri Lanka (CPSL) aus dem Kabinett ausgeschieden. Mit 139 von insgesamt 166 Parlamentssitzen verfügte die UNP nun über eine verfassungsändernde Mehrheit.

In dieser Lage besannen sich die Oppositionsparteien ihrer gewerkschaftlichen Vorfeldorganisationen als außerparlamentarischer Kraft. Die Regierung verfügte als Gegengewicht über eigene Gewerkschaften, mit denen sie außerdem die Kalkulierbarkeit des Faktors Arbeit für ihre neue Wirtschaftspolitik sichern wollte.

Unabhängig-oppositionelle, berufsständische und von Minderheiten dominierte Gewerkschaften existierten als weitere Faktoren zwischen diesen Parteiblöcken. Bei gewerkschaftlichen und politischen Auseinandersetzungen kam es deshalb zu wechselnden Bündniskonstellationen. Der ungelöste singhalesisch-tamilische Konflikt hatte dabei eine besondere Bedeutung.

Im folgenden werden zunächst die Strukturen der Gewerkschaftsbewegung und das Phänomen der politischen Patronage analysiert. Vor diesem Hintergrund wird auf den Streik im Juli 1980, das antitamilische Pogrom im Juli 1983 und den Plantagenarbeiterstreik 1984 eingegangen. Neuere Entwicklungen zeigen Variationen in der Zusammenarbeit von Gewerkschaften bei relativ stabilen Grundstrukturen. Das politische und wirtschaftliche Umfeld insgesamt ist für die Gewerkschaften schwieriger geworden.

\section{Strukturmerkmale}

\subsection{Differenzierte Vielfalt}

Im Register des Arbeitsministeriums sind etwa 1000 Gewerkschaften gemeldet. Diese Vielfalt erschwert die Analyse und Beurteilung von gewerkschaftlichen Entwicklungen in Sri Lanka. Deshalb werden für die weitere Analyse die Gerwerkschaftstypen Sri Lankas anhand folgender Kriterien unterteilt: Kontrolle durch Parteien; unabhängig-opposi- 
tionelle Orientierung; Vertretung ethnischer Minderheiten; berufsständische Orientierung. Daneben wird die internationale Orientierung berücksichtigt. In den feingegliederten gewerkschaftlichen Strukturen bestätigt sich die ethnische, politische, soziale, religiöse und wirtschaftliche Differenziertheit des Inselstaates.

Viele registrierte Gewerkschaften nehmen kaum Funktionen wahr. Manche existieren nur auf dem Papier. Diese "Papiergewerkschaften" sind die Ergebnisse gescheiterter Spaltungsversuche, des Bedeutungsverlustes durch politischen Wandel oder des Wunsches exzentrischer Persönlichkeiten, Präsident einer Organisation zu sein. Die Gewerkschaftsgesetzgebung stellt geringe Anforderungen an die Registrierung. Im öffentlichen Dienst erzwingt sie eine enge horizontale Abgrenzung durch restriktive Vorschriften über den zulässigen Organisationsbereich. ${ }^{1}$

Die extreme Zersplitterung empfinden viele Gewerkschafter als großes Problem und Hauptursache der strukturellen Schwäche der gesamten Bewegung. Die Folge ist ein selbst für asiatische Verhältnisse sehr niedriges Lohnniveau und Stagnation in der sozialen Entwicklung.

\subsection{Parteigewerkschaften}

Ein wesentlicher Teil der Gewerkschaftsbewegung wird durch politische Parteien kontrolliert. ${ }^{2}$ Alle Parteien, die seit 1970 an Regierungen beteiligt waren, üben Einfluß auf eigene parteinahe Gewerkschaften aus:

1 Sec. 21(1)b Trade Union Ordinance.

2 Der Anteil lag um 1970 bei über einem Viertel (R. N. Kearney, Trade Unions and Politics in Ceylon, (1971)). Nach Schätzungen von Vertretern des Arbeitsministeriums stieg der Anteil nach dem UNP Wahlsieg weiter an. 


\begin{tabular}{|c|c|c|}
\hline Partei & Gew. Dachverband & Organisationsbereich \\
\hline $\begin{array}{l}\text { United } \\
\text { National }\end{array}$ & Jathika Sevaka Sangamaya (JSS) & Privatsektor u. Staatsbetriebe \\
\hline Party & $\begin{array}{l}\text { Lanka Jathika Estate } \\
\text { Workers Union (LJEWU) }\end{array}$ & Plantagen \\
\hline (UNP) & $\begin{array}{l}\text { Public Service National Trade } \\
\text { Union Federation (PSNTUF) }\end{array}$ & Offentlicher Dienst \\
\hline $\begin{array}{l}\text { Sri Lanka } \\
\text { Freedom } \\
\text { Party } \\
\text { (SLFP) }\end{array}$ & $\begin{array}{l}\text { Sri Lanka Independent Trade } \\
\text { Union Federation (SLITUF) } \\
\text { Sri Lanka Independent } \\
\text { Government } \\
\text { Trade Union Federation (SLIGTUF }\end{array}$ & Privatsektor u. Staatsbetriebe \\
\hline $\begin{array}{l}\text { Communist } \\
\text { Party of } \\
\text { Sri Lanka } \\
\text { (CPSL) }\end{array}$ & $\begin{array}{l}\text { Ceylon Federation of } \\
\text { Trade Unions (CFTU) } \\
\text { Public Service Trade Union } \\
\text { Federation (PSTUF) }\end{array}$ & $\begin{array}{l}\text { Privatsektor u. Staatsbetriebe } \\
\text { Offentlicher Dienst }\end{array}$ \\
\hline $\begin{array}{l}\text { Lanka } \\
\text { Samasamaja } \\
\text { Party }\end{array}$ & $\begin{array}{l}\text { Ceylon Federation of } \\
\text { Labor (CFL) }\end{array}$ & Privatsektor u. Staatsbetriebe \\
\hline (LSSP) & $\begin{array}{l}\text { Government Worker's Trade } \\
\text { Union Federation (GWTUF) }\end{array}$ & Offentlicher Dienst \\
\hline
\end{tabular}




\begin{abstract}
Mitglieder Internationaler Gewerkschaftsorganisationen (Auswahl)*
Internationale $^{14}$ Mitgliedsorganisation Klassifikation (Sitz)
\end{abstract}

\begin{tabular}{lll}
\hline WFTU & $\begin{array}{l}\text { Ceylon Federation of Trade Unions } \\
\text { (Prag) }\end{array}$ & $\begin{array}{l}\text { Parteigewerkschaft (CPSL) } \\
\text { Public Service Trade Union }\end{array}$ \\
& $\begin{array}{l}\text { Federation } \\
\text { Sri Lanka Independent Trade }\end{array}$ & Parteigewerkschaft (SLFP) \\
& $\begin{array}{l}\text { Union Federation } \\
\text { Sri Lanka Independent Goverment }\end{array}$ & Parteigewerkschaft (SLFP) \\
& $\begin{array}{l}\text { Trade Union Federation } \\
\text { Democratic Workers Congress }\end{array}$ & oppositionell \\
& Ceylon Workers Congress & ethnisch (Indien-Tamilen) \\
\hline ICFTU & & \\
\hline Brüssel) & &
\end{tabular}

\begin{tabular}{lll}
\hline $\begin{array}{l}\text { PTTI } \\
\text { (Genf) }\end{array}$ & $\begin{array}{l}\text { Union of Post \& Telecom. Officers } \\
\text { Telecommunication Officers Union }\end{array}$ & $\begin{array}{l}\text { berufsständisch } \\
\text { berufsständisch }\end{array}$ \\
\hline $\begin{array}{l}\text { IMF } \\
\text { (Genf) }\end{array}$ & Jathika Sevaka Sangamaya & Parteigewerkschaft (UNP) \\
\hline
\end{tabular}

\begin{tabular}{lll}
\hline $\begin{array}{l}\text { IFBWW } \\
\text { (Genf) }\end{array}$ & Jathika Sevaka Sangamaya & Parteigewerkschaft (UNP) \\
\hline $\begin{array}{l}\text { IFPAAW } \\
\text { (Genf) }\end{array}$ & $\begin{array}{l}\text { Ceylon Workers Congress } \\
\text { Lanka Jathika Estate Workers } \\
\text { Union }\end{array}$ & ethnisch (Indien-Tamilen) \\
\hline
\end{tabular}

\begin{tabular}{lll}
\hline IUF & Ceylon Mercantile Industries \& & \\
(Genf) & General Workers Union & unabh.-oppositionell \\
& Ceylon Estates Staff Union & $\begin{array}{l}\text { unabh.-oppositionell } \\
\text { oppositionell }\end{array}$ \\
\hline
\end{tabular}

\begin{tabular}{lll}
$\begin{array}{l}\text { IFFTU } \\
\text { (Amsterdam) }\end{array}$ & $\begin{array}{l}\text { Ceylon Tamil Teachers Union } \\
\text { All Ceylon Goverment English } \\
\text { Teachers Union }\end{array}$ & ethnisch (Sri Lanka Tamilen) \\
\hline $\begin{array}{l}\text { PSI } \\
\text { (Genf) }\end{array}$ & $\begin{array}{l}\text { Ceylon Workers Congress } \\
\text { S.L.P.H.S.S. }\end{array}$ & $\begin{array}{l}\text { ethnisch (Indien-Tamilen) } \\
\text { parteinah (SLFP) }\end{array}$ \\
\hline
\end{tabular}

ITGLF Ceylon Mercantile, Industries \& unabh.-oppositionell

(Brüssel) General Workers Union

* Tabelle zu 2.6, S. 143 
Starke innerparteiliche Konflikte oder Parteispaltungen wirken sich auch auf die Parteigewerkschaften aus. Nachdem sich im Jahre 1983 die SLMP (Sri Lanka Mahajana Party) von der SLFP (Sri Lanka Freedom Party) abspaltete, entwickelten sich entsprechende gewerkschaftliche Flügel, die später auseinanderbrachen.

\subsection{Parteiunabhängige, oppositionelle Gewerkschaften}

Mit knapp 40000 Mitgliedern ist die Ceylon Mercantile, Industries and General Workers Union $(\mathrm{CMU})^{3}$ die stärkste Gewerkschaft dieser Kategorie. Weitere bedeutende Organisationen sind The Ceylon Bank Employees Union (CBEU) und die Ceylon Estates Staffs Union (CESU).

Diese Gewerkschaften sind parteiunabhängig, haben aber dennoch ein politisches Selbstverständnis. Neben der ökonomischen Interessenvertretung ihrer Mitglieder streben sie auch die Umgestaltung der Gesellschaft mit sozialistischer Perspektive an. Gewerkschaftliche Gegenmacht entwickeln sie auf der Basis guter betrieblicher Verankerung und straffer Organisation. Damit unterscheiden sie sich von den parteipolitisch kontrollierten Gewerkschaften. ${ }^{4}$ Unter schwierigen rechtlichen politischen Rahmenbedingungen erkämpften sie ökonomische Vorteile für ihre Mitglieder. Dies bestätigen die erfolgreich abgeschlossenen Tarifverträge. ${ }^{5}$ Ihr Organisationsschwerpunkt sind die Beschäftigten des urban-privatwirtschaftlichen Bereichs, in dem sich politische Patronage nicht so leicht entfalten kann. Unter ihren Mitgliedern finden sich Anhänger aller Parteien und Vertreter verschiedener ethnischer Gruppen. Durch ihre parteipolitische Unabhängigkeit und starke betriebliche Verankerung überstanden diese Gewerkschaften alle Regierungswechsel ohne größere Einbrüche.

\subsection{Gewerkschaften ethnischer Minderheiten}

Die offizielle Statistik teilt die Bevölkerung Sri Lankas in folgende ethnische Gruppen ein: 6

$\begin{array}{ll}\text { Singhalesen } & (71,9 \%) \\ \text { Ceylon Tamilen } & (11,9 \%) \\ \text { Indien Tamilen } & (9,4 \%) \\ \text { Moors } & (6,7 \%) \\ \text { Sonstige } & (0,9 \%)\end{array}$

In einigen Beschäftigungsbereichen dominieren ethnische Minderheiten. Auf diesem Feld agieren Gewerkschaften, die ihre Mitglieder nicht nur als Arbeitnehmer, sondern

3 Bis 1984: Ceylon Mercantile Union (CMU).

4 Einige CFTU Gewerkschaften nehmen hier eine Zwischenstellung ein. Sie werden zwar von der CPSL kontrolliert, verfügen aber aufgrund guter betrieblicher Verankerung über autonome gewerkschaftliche Gegenmacht.

5 Vgl.: P. Navaratne, Collective Agreements in Sri Lanka, (1984), S. $152 \mathrm{ff}$.

6 Demographic Atlas of Sri Lanka, (1980).

7 Moslemische, überwiegend tamilischsprachige Bevölkerungsgruppe. 
auch als Angehörige einer ethnischen Minderheit vertreten. In Extremsituationen können die gewerkschaftsspezifischen Aufgaben gegenüber dieser Funktion sogar vorübergehend zurücktreten. ${ }^{8}$

Die Ceylon Tamil Teachers Union (CTTU) organisiert knapp 10000 Ceylon Tamilen und tamilisch sprechende Moors. Ihre Mitglieder sind, überwiegend im tamilischsprachigen Norden und Osten der Insel, als Lehrer tätig. Politisch steht die CTTU den nichtmilitanten Separatisten in der Tamil United Liberation Front (TULF) nahe. Seit sich die innenpolitische Situation ab 1983 ständig verschlechtert hat, findet an vielen tamilischen Schulen nur noch unregelmäßig Unterricht statt. Die Aktivitäten der CTTU verlagerten sich seitdem immer stärker auf die Vertretung der Lehrer in der Minderheitenfrage. Ab 1985 kontrollierten die militanten Separatisten Teile des Nordens um Jaffna. In dieser Situation mußte die CTTU eine schwierige Gratwanderung zwischen Duldung durch die Separatisten im Norden und Zusammenarbeit mit dem Erziehungsministerium in Colombo gehen. Die Verschlechterung der Sicherheitssituation erschwerte die Gewerkschaftsarbeit der CTTU in der jüngsten Vergangenheit zunehmend.

Der Ceylon Workers Congress (CWC) ist mit über 250000 Mitgliedern die stärkste Gewerkschaft Sri Lankas. Gewerkschaftliche Basis des CWC sind die tamilischen Plantagenarbeiter der Teeanbaugebiete im zentralen Hochland. Diese Indien-Tamilen wurden seit etwa 1880 im südindischen Tamil Nadu als Arbeiter für die expandierende Plantagenwirtschaft Sri Lankas angeworben. ${ }^{9}$ Noch heute konzentrieren sich die Indien-Tamilen auf die Teeanbaugebiete. Nach der Unabhängigkeit Ceylons wurden viele tamilische Plantagenarbeiter staatenlos. ${ }^{10}$ Der CWC konzentrierte sich deshalb neben seiner gewerkschaftlichen Arbeit stets auch auf die Lösung der politischen und ethnischen Probleme der Plantagenarbeiter.

Der CWC betätigt sich in Form eines 'politischen Flügels ${ }^{11}$ auch als politische Partei. 1977 eroberte er im Wahlkreis Nuwara Eliya ein Direktmandat im Parlament. Seit 1978 beteiligt er sich in einer Art Koalition an der UNP-Regierung. Präsident S. Thondaman hält im gegenwärtigen Kabinett das Ressort 'Rural Industrial Development‘.

Seit einigen Jahren bemüht sich der CWC mit mäßigem Erfolg verstärkt um die Aufnahme nichttamilischer Mitglieder. Dazu gibt er seit kurzem auch eine singhalesische Mitgliederzeitung heraus. Dennoch hat dies das Selbstverständnis des CWC und von

8 Dies geschah z.B. nach den Ereignissen im Juli 1983. Tamilische Gewerkschaften kümmerten sich um die Versorgung in Flüchtlingslagern und die Betreuung betroffener Familien.

9 Damit unter den harten Arbeitsbedingungen bei niedrigem Lohnniveau der Betrieb auf den Plantagen aufrechterhalten werden konnte, wurden Arbeitskräfte benötigt, die faktisch nicht über Beschäftigungsalternativen verfügten. Die ortsansässigen singhalesischen Kleinbauern konnten ausweichen und sich in die Subsistenzwirtschaft zurückziehen. Den meist mittellosen südindischen Arbeitern war diese Möglichkeit in der fremden Umgebung verschlossen.

Vgl.: D. Wesumperuma, Indian Immigrant Plantation Workers in Sri Lanka, (1986).

10 Vgl.: L.L.T. Peiris, The Citizenship Law of the Republic of Sri Lanka (Ceylon), (1974).

11 Sec. 47 Trade Union Ordinance gestattet Gewerkschaften außerhalb des öffentlichen Dienstes die Verfolgung politischer Ziele. Diese Aktivitäten müssen durch einen separaten "political fund" finanziell abgegrenzt werden. 
Minister S. Thondaman als gewerkschaftliche und politische Repräsentanten der Tamilen indischer Herkunft nicht grundlegend verändert. ${ }^{12}$

\subsection{Berufsständische Gewerkschaften}

Eine größere Zahl recht kleiner Gewerkschaften konzentriert sich auf eine bestimmte Hierarchieebene innerhalb eines eng begrenzten Fachgebietes. Diese Gewerkschaften agieren vor allem im öffentlichen Dienst. Gegenüber Arbeitgeber und Gesellschaft vertreten sie die berufsständischen Interessen ihrer Mitglieder. Sie halten sich politisch zurück und versuchen, mit der jeweiligen Regierung gut zurechtzukommen. Gefahr droht ihnen, wenn regierungsnahe Parteigewerkschaften versuchen, in ihren Organisationsbereich einzubrechen. In dieser Situation haben einige eine pragmatische Verteidigungsstrategie entwickelt: Sie lassen Doppelmitgliedschaften zu. Als Zusatzversicherung treten ihre Mitglieder gegen geringen Beitrag nebenbei den jeweiligen Regierungsgewerkschaften bei. Ihre authentische Interessenvertretung sehen diese Arbeitnehmer aber weiterhin in ihrer berufsständischen, parteiunabhängigen Gewerkschaft.

Die Union of Post \& Telecommunication Officers (UPTO) ist mit ca. 4000 Mitgliedern eine der größten berufsorientierten Gewerkschaften. Sie unterscheidet sich von ihren kleineren Schwestergewerkschaften insofern, als sie stets Kontakte zur politisch orientierten Gewerkschaftsbewegung aufrecht hielt. An gemeinsamen Protestaktionen dieser Gewerkschaften gegen die Regierung beteiligte sie sich jedoch im allgemeinen nicht. Privatisierungsabsichten im Postbereich vergrößerten in der jüngeren Vergangenheit ihre Distanz zur Regierung. Seitdem kam es zu einer Annäherung an die parteiunabhängigen oppositionellen Gewerkschaften.

Weitere kleinere Gewerkschaften, die Berufsgruppen im öffentlichen Dienst organisieren, sind z. B.: Government Surveyors' Union; Government X-Ray Technical Officers' Union; Public Health Insepctors' Union; All Ceylon Government English Teachers Union; Sri Lanka Accountant Service Association; Labour Officers' Association u.v.m.

\subsection{Internationale Orientierung}

Die Mitgliedschaft in internationalen Dachverbänden und Berufssekretariaten (IBS) ist für Gewerkschaften in Entwicklungsländern von größerer Bedeutung als in Industrieländern. Die Internationalen unterstützen ihre Mitglieder in Entwicklungsländern durch die Finanzierung von Bildungsprogrammen und Entwicklungsprojekten. ${ }^{13}$ Sie ermöglichen die Teilnahme von Funktionären an internationalen Kongressen und sichern dadurch den internationalen Informationsfluß. Die Hilfe kann einen wesentlichen Teil des

12 Vgl. hierzu: The Plantation Worker - a Voice in a United Sri Lanka, CWC (1986).

13 Der CWC und seine gewerkschaftliche Stiftung CLF erhalten z.B. Unterstützung von IFPAAW, ICFTU, der norwegischen Entwicklungshilfeorganisation NORAD und der deutschen Gewerkschaft Gartenbau, Landund Forstwirtschaft. 
Gewerkschaftsbudgets ausmachen; in manchen Fällen übersteigt sie den aus eigener Kraft erbrachten Budgetanteil.

Einziges Mitglied des Internationalen Gewerkschaftsbundes ICFTU, dem auch die wichtigsten westeuropäischen Gewerkschaften angehören, ist der CWC. Trotz intensiver Bemühungen gelang den UNP-Gewerkschaften der Beitritt zum ICFTU nicht. Die zahlreichen IBS organisieren eine große Bandbreite von Gewerkschaften. Ihre Bedeutung hat seit 1977 zugenommen. Die IBS stehen dem ICFTU nahe, sind aber unabhängig. Dem osteuropäisch dominierten WFTU gehören die Parteigewerkschaften von CPSL und SLFP und der vom CWC abgespaltene DWC an (s. Tab., S. 140).

\section{Politische Patronage}

Die marxistischen Parteien sahen in Gewerkschaften die natürlichen Organisationen der Arbeiterklasse. Sie benötigten den Zugang zu Gewerkschaften, um im leninschen Sinne die Transmission zwischen Partei und Arbeiterklasse sicherzustellen. ${ }^{15}$

Mit marxistisch-leninistischen Konzeptionen alleine läßt sich die Existenz parteipolitisch kontrollierter Gewerkschaften in Sri Lanka jedoch nicht hinreichend erklären. Alle nennenswerten nicht-marxistischen Parteien verfügen ebenfalls über eigene, von ihnen kontrollierte Gewerkschaften. Einige davon üben im Bereich der an- und ungelernten Arbeiter größeren Einfluß aus.

Im Gegensatz zur Mitgliedschaft repräsentiert die Führung dieser Gewerkschaften meist die politische, gelegentlich auch die wirtschaftliche Elite des Landes. In seiner Analyse über Gewerkschaften und Politik in Ceylon untersuchte Kearney Ende der 60er Jahre das Phänomen der organisatorischen Verbindung von Politikern der Elite mit Arbeitern in Gewerkschaften. Bei den Parteiführern sieht er das Interesse am Zugang zu den Massen: ${ }^{16}$

"The partisan function of a labor organization of which politicians are most conscious is that of a channel to "the people" or "the masses", a link between the party and a relatively broad segment of the population which is not effectively reached by the party's own organization. Trade

Vollständige Bezeichnungen (s. Tab., S. 140):

WFTU - World Federation of Trade Unions

ICFTU - International Confederation of Free Trade Unions

PTTI - Postal, Telegraph and Telephone International

IMF - International Metalworkers' Federation

IFBWW - International Federation of Building and Woodworkers

IFPAAW - International Federation of Plantation, Agriculture and Allied Workers

IUF - International Union of Ford and Allied Workers' Associations

IFFTU - International Federation of Free Teachers Union

PSI _ - Public Services International

ITGLF - International Textile, Garment and Leather Workers' Federation.

15 Vgl.: R. N. Kearny, Fn. 2, S. 62

16 R. N. Kearny, Fn. 2, S. 68. 
unions and youth leagues are the two types of mass organizations employed by most Ceylonese parties in the attempt to project their influence beyond the narrow and predominately middleclass membership of the party."

Die Motive der Arbeiter erklärt er u.a. mit den traditionellen paternalistischen Gesellschaftsstrukturen Sri Lankas: ${ }^{17}$

"The desire of workers to place themselves under the protection of a powerful guardian may be related to the patron-client relations of traditional society and the distrust and insecurity prevalent in the culture. This may be one reason why the formal head of a party's trade union center is usually one of the most prominent top party leaders, although he is presumably seldom able to devote much time and attention to union matters."

In der europäischen Gewerkschaftstradition sind identische Klasseninteressen Grundlage der solidarischen Zusammenarbeit in Gewerkschaften. In Sri Lanka ist in den Gewerkschaften der nicht-marxistischen Parteien UNP und SLFP eine wirtschaftliche und soziale Interessenidentität von Führung und Basis nicht oder nur sehr begrenzt vorhanden. Gewerkschaftliche Solidarität kann deshalb nicht Grundlage des vertikalen Zusammenhalts dieser Organisationen sein. Der Zusammenhalt wird durch eine Warentauschbeziehung zwischen Arbeitern und Politikern, vor dem Hintergrund unterschiedlicher Interessen und auf der Grundlage paternalistischer Gesellschaftsstrukturen gewährleistet. Der Tausch ist vorteilhaft, wenn jeder der Beteiligten auf 'komperative Kostenvorteiler verweisen kann. Welche 'Waren' werden zu welchen 'terms of trade' getauscht?

Der Politiker tauscht bei diesem Handel ein: Zugang zu Bevölkerungsschichten; Massenunterstützung bei Veranstaltungen und Wahlkampagnen; Einfluß auf Wirtschaftszweige; eine stärkere Position in der Partei; Hilfstruppen für verschiedene Aufgaben des politischen Tagesgeschäfts. Demgegenüber erhoffen sich die Arbeiter: Statusgewinn und Sicherheit durch politische Patronage; politische Unterstützung bei der Durchsetzung ihrer Forderungen; Teilhabe an Macht und Einfluß des Politikers; Gewogenheit staatlicher Stellen.

Diese Beziehung erklärt, wieso Minister aus einflußreichen Familien und Arbeiter der ärmsten Bevölkerungsgruppen sich gemeinsam in einer Gewerkschaft wiederfinden. Geprägt durch die paternalistisch-hierarchische Struktur der ceylonesischen Gesellschaft legen die Arbeiter ihr Anliegen in die Hände eines politischen Führers aus der Elite. Dafür gewähren sie ihm, zumindest zeitlich begrenzt, ihre Loyalität und Unterstützung. Wegen der hierarchischen Struktur besteht in dieser Beziehung ein Machtgefälle. Die Arbeiter müssen deshalb befürchten, daß der politische Führer ihre ursprünglichen Anliegen entsprechend seinen Interessen modifiziert. Der kulturelle Rahmen läßt eine offene innergewerkschaftliche Auseinandersetzung mit der politischen Führung nicht zu. Nachdem die Arbeiter dieses Bündnis eingegangen sind, haben sie unter gegebenen politischen Rahmenbedingungen kaum noch die Möglichkeit, es wieder zu beenden.

Diese Beziehung gerät jedoch ins Wanken, wenn sich das politische Umfeld wandelt. 
Personelle Auswirkungen auf eine Parteigewerkschaft haben z. B. fundamentale Änderungen in der Machtposition der Partei oder des Politikers:

Die Partei des Politikers verliert die Wahlen und die Regierung wechselt. Damit verliert der Politiker seine Attraktivität für die Arbeiter. Wegen der hierarchischen Strukturen in den politisch kontrollierten Gewerkschaften werden die Arbeiter nicht die Möglichkeit haben, ihren Führer gegen einen neuen, mächtigeren auszuwechseln. Die Arbeiter verlassen statt dessen ihre bisherige Gewerkschaft und treten einer neuen bei. Nur ein enger Kreis von Funktionsträgern und Parteianhängern wird bei der alten Gewerkschaft bleiben und dort auf bessere Zeiten warten. Dieser Prozeß fand 1977 nach der Wahlniederlage der SLFP-Regierung von Frau Banderanaike statt. Die bis dahin einflußreichen SLFP-Gewerkschaften verloren einen Großteil ihrer Mitglieder; die UNP-Gewerkschaften gewannen entsprechend.

Ein anderer Ablauf tritt ein, wenn die Partei ihre Macht behält, der Politiker aber an Bedeutung verliert. Der Politiker wird die von ihm erwartete Rolle dann nicht mehr ausfüllen können. Damit verlieren Partei und Arbeiter ihr Interesse an ihm. Dieser Fall trat 1985 ein, als JSS-Präsident Cyril Mathews das Kabinett verlassen mußte. Der bisherige Industrie- und Forschungsminister wurde kurz darauf auch seines Präsidentenamtes bei JSS entkleidet. Da sich die UNP über eine endgültige Nachfolge noch nicht einigen konnte, erhielten Sicherheitsminister Lalith Athulathmudali und Erziehungsminister Ranil Wickremasinghe Vizepräsidentenämter. Um die endgültige Entscheidung über die politische Führung offenzuhalten, wurde vorerst der politisch bedeutungslose Neville Perera zum Präsidenten bestimmt.

Diese Zusammenhänge gelten nicht für politisierte Gewerkschaften wie CMU oder CWC, deren Macht auf betrieblicher Organisation basiert. Politisierung unterscheidet sich von parteipolitischer Patronage. CMU und CWC konnten deshalb alle Regierungswechsel relativ gut überstehen.

\section{Der "Juli '80-Streik"}

\subsection{Vorgeschichte und Ablauf}

Im Januar 1978 fanden sich Gewerkschaften der Oppositionsparteien und unabhängigoppositionelle Gewerkschaften zu einem Aktionsbündnis zusammen. Dem 'Joint Trade Union Action Committeer (JTUAC) gehörten folgende 16 Organisationen an:

Ceylon Federation of Trade Unions (CFTU)

Ceylon Federation of Labour (CFL)

Public Service Trade Union Federation (PSTUF)

Government Clerical Sercice Union (GCSU)

United Federation of Labour (UFL)

Samastha Lanka Rajaye Lipikaru Sangamaya (SLRLS)

Sri Lanka Independent Trade Union Federation (SLITUF) 
Sri Lanka Jathika Guru Sangamaya (SLJGS)

Government Worker's Trade Union Federation (GWTUF)

Sri Lanka Independent Government Trade Union Federation (SLIGTUF)

Local Government Trade Union Federation (LGTUF)

Ceylon Trade Union Federation (CTUF)

Ceylon Mercantile Union (CMU)

Ceylon Bank Employees Union (CBEU)

Ceylon Estates Staffs Union (CESU)

Technical Services Union Federation (TSUF)

Dieses Bündnis sollte die Aktivitäten der oppositionellen Gewerkschaften koordinieren, um gemeinsame Forderungen effektiver durchzusetzen. Am 8. und 9. März 1980 hielt das JTUAC eine Konferenz ab, auf der ein gemeinsamer Forderungskatalog beschlossen wurde. Neben der Forderung nach Lohnerhöhungen wurden auch Beschlüsse zur Verteidigung von Gewerkschaftsrechten gefaßt. Am 5. Juni 1980 sollte ein 'Nationaler Tag des Protestes'gegen arbeitnehmerfeindliche Maßnahmen der UNP-Regierung stattfinden.

Diesem Aufruf folgten auch die gut organisierten Arbeiter des 'Ratmalana Railway Workshop', südlich von Colombo. Ein Proteststreik am 5. Juni mündete in den folgenden Wochen in eine Serie betrieblicher Auseinandersetzungen. Die Betriebsleitung suspendierte daraufhin am Freitag, dem 4. Juli 1980, 12 der beteiligten Arbeiter. Noch am gleichen Tag kam es zu einer spontanen Arbeitsniederlegung, die am Montag, den 7. Juli, fortgesetzt wurde und sich in den Tagen darauf im Bereich der staatlichen Eisenbahnen ausweitete.

Das JTUAC trat am 13. Juli zusammen, um die Lage nach dieser spontanen Entwicklung zu beraten. 12 Gewerkschaften beschlossen zum 14. Juli, einen Generalstreik auszurufen. In einem Schreiben an Staatspräsident J. R. Jayewardene wurde mitgeteilt: ${ }^{18}$

"Sehr geehrter Herr Präsident, anläßlich einer Sitzung der JTUAC haben die unten erwähnten Gewerkschaften gestern beschlossen, einen Generalstreik auszurufen mit dem Ziel, folgende Forderungen durchzusetzen:

1) Gewährung einer sofortigen Lohnerhöhung von Rs. 300.-

2) Sofortige Lohnerhöhung auf Basis von Rs. 5.- - für jeden Punkt des Colombo Konsumenten Preisindex vom Juli 1977 bis heute und eine weitere Erhöhung von Rs. 5.- / Punkt für künftige Erhöhungen des Lebenskostenindex.

3) Straferlaß für alle, die an der Protestbewegung vom 5. Juni 1980 teilgenommen haben."

Die gut organisierten, parteiunabhängigen Gewerkschaften CMU, CBEU und CESU unterstützten diesen Aufruf nicht. Sie hielten den Streikaufruf für überstürzt und wegen mangelnder Erfolgsaussichten für unverantwortlich.

1812 JTUAC organisations to President J. R. Jayewarene on 14th July 1980, (Ubers. a. d. Englischen) aus: July 1980 Strike, Dokumente und Stellungnahmen, unveröffentlicht (o.D.). 
Die Regierung konterte von Anfang an hart. Sie erklärte den Ausnahmezustand. Damit waren alle öffentlichen Veranstaltungen im Zusammenhang mit dem Streik illegal. Die Regierung konnte nun auch die Bankkonten der beteiligten Gewerkschaften vorübergehend sperren lassen. Schließlich stellte sie den Streikenden als Ultimatum, bis spätestens 18. Juli wieder an die Arbeit zu gehen. ${ }^{19}$ Anderenfalls würde davon ausgegangen, daß die Betroffenen ihre Arbeitsplätze verlassen hätten und an einer Beschäftigung nicht mehr interessiert seien.

Diese Drohung wirkte. Ab 18. Juli begann die ohnehin geringe Beteiligung ${ }^{20}$ abzubrökkeln. Als dies sichtbar wurde, machte die Regierung ihre Drohung wahr und betrachtete die Arbeitsverträge der immer noch Streikenden als aufgelöst. Auf einem Treffen der 12 streikenden Gewerkschaften a m 22. August lagen die Namen von 28000 Beschäftigten vor, die ihren Arbeitsplatz verloren hatten. ${ }^{21}$

\subsection{Auswirkungen auf die Gewerkschaftsbewegung}

Nach der Regierungsübernahme war die UNP-Regierung bestrebt, Kontrolle über die Aktionen der organisierten Arbeitnehmerschaft zu erlangen. Pate stand bei diesen Uberlegungen das Verhältnis von Regierung und Gewerkschaften in Singapur. Regierungskonforme Gewerkschaften sollten sicherstellen, daß die Rolle des Faktors Arbeit in Entwicklungsplanung und Wirtschaftspolitik risikolos kalkulierbar wird.

Die gewerkschaftlichen Vorfeldorganisationen der UNP (JSS, LJEWU, PSNTUF) hatten nach dem Wahlsieg einen starken Mitgliederzuwachs zu Lasten der SLFP-Gewerkschaften. Kraft und Einfluß dieser Organisationen reichten jedoch nicht aus, um die geplante Rolle wirksam zu übernehmen. Die UNP war deshalb auf die Zusammenarbeit mit dem CWC angewiesen. Der CWC wiederum sah im Bündnis mit der UNP eine gute Voraussetzung zur Durchsetzung der außergewerkschaftlichen Interessen seiner tamilischen Mitgliedschaft. Damit konnte der CWC vorübergehend in die Gewerkschaftsstrategie der UNP integriert werden.

Als regierungsunabhängige und kampfbereite gewerkschaftliche Kräfte waren nur die unabhängig-oppositionellen Gewerkschaften und die nach dem Regierungswechsel an Mitgliedern geschwächten Gewerkschaften der Oppositionsparteien verblieben. Vor diesem Hintergrund schätzten die parteiunabhängigen Oppositionsgewerkschaften CMU, CBEU und CESU das Kräfteverhältnis zwischen Gewerkschaften und Regierung realistisch ein. Deshalb unterstützten sie den Streikaufruf nicht:22

"None of the three unions ultimately joined the strike, in the context of its manifest ineffectiveness and the anti-strike measures of the Government under the Emergency, including especially the preventions of meetings in preparation for strike action."

19 Als rechtliche Grundlage diente die 'Essential Services Order'.

20 Die beteiligten Gewerkschaften nennen ca. 100000 Streikende. Beobachter nicht streikender Gewerkschaften schätzen die Beteiligung auf 40000 .

21 Vgl.: July 1980 Strike, Fn. 18, S. 46.

22 CMU Gen. Sec. B. Tampoe, Statement presented to CMU Delegates' Conference Sept. 19-21, 1980, in: July 1980 Strike, Fn. 18, S. 43. 
Die 12 streikenden Gewerkschaften waren durchweg von Oppositionsparteien kontrolliert oder zumindest beeinflußt:

\section{Gewerkschaft Partei}

$\begin{array}{ll}\text { CFTU } & \text { CPSL (Communist Party of Sri Lanka) } \\ \text { CFL } & \text { LSSP (Lanka Samasamaja Party) } \\ \text { PSTUF } & \text { CPSL } \\ \text { GCSU } & \text { NSSP (Nava Sama Samaja Party) } \\ \text { UFL } & \text { NSSP } \\ \text { SLRLS } & \text { CPSL } \\ \text { SLITUF } & \text { SLFP (Sri Lanka Freedom Party) } \\ \text { SLJGS } & \text { CPSL } \\ \text { GWTUF } & \text { LSSP } \\ \text { SLIGTUF } & \text { SLFP } \\ \text { LGTUF } & \text { NSSP u. LSSP beeinflußt } \\ \text { CTUF } & \text { CCP (Ceylon Communist Party) }\end{array}$

Die Parteien hinter diesen Gewerkschaften erhofften sich aus dem Streik eine Stärkung ihrer außerparlamentarischen Position. Diese Hoffnung erfüllte sich nicht. Sie kehrte sich ins Gegenteil. Die Massenbeteiligung blieb aus. Die Gegenmaßnahmen der Regierung erwiesen sich als wirksam. Durch die Niederlage wurde die Position der linken Parteigewerkschaften ein weiteres Mal nach dem Regierungswechsel empfindlich geschwächt. Ihre betriebliche Organisation wurde durch die Entlassung von $28000 \mathrm{der}$ treuesten Anhänger über weite Bereiche aufgelöst. Viele der vakanten Positionen konnten mit Anhängern der Regierung besetzt werden. Dies stärkte die Position der UNPGewerkschaften.

Die CMU bewertet den 'Juli '80-Streik' der parteikontrollierten Oppositionsgewerkschaften folgerichtig als ernsten Rückschlag für die Gewerkschaftsbewegung des Landes: ${ }^{23}$

"This Delegates' Conference recognises, however, that the disastrous failure of the strike called jointly by twelve organisations of the JTUAC on 14th July, has resulted in a severe setback for the organized working class, besides seriously damaging if not wrecking the JTUAC as an effective trade union centre."

Die Ereignisse um den Streik mündeten schließlich noch auf einem ganz anderen Schauplatz in eine gewerkschaftliche Auseinandersetzung. Das Verhältnis von CMU und CWC war nach der CWC-Regierungsbeteiligung zunehmend abgekühlt. Beide Organisationen gehörten dem Berufssekretariat International Union of Food and Allied Workers Association' (IUF) an. Die CMU hatte sich zwar selbst nicht aktiv am Streik betei-

23 Resolution Adopted at 16th Delegates' Conference, in: July 1980 Strike, Fn. 18, S. 48. 
ligt, betrieb nun aber den Ausschluß des CWC aus IUF, da Präsident Thondaman als Kabinettsminister die Verantwortung für die Entlassung der Streikenden mittrage. CMU-Generalsekretär Tampoe setzte sich durch und der CWC wurde ausgeschlossen. Als Folge verließen nun auch die singapurianischen Mitgliedsorganisationen IUF. Das IUF-Regionalbüro in Singapur mußte deshalb nach Australien verlegt werden.

Ergebnis des Streiks und der Ausschlußaktion war, daß sich die Kluft zwischen CWC und Oppositionsgewerkschaften vertiefte. Die UNP-Gewerkschaften erreichten nun den Höhepunkt ihrer Bedeutung. Die Gewerkschaften der Oppositionsparteien waren nach dem Streik über weite Bereiche aufgelöst. Autonome gewerkschaftliche Kraft repräsentierten vorübergehend nur noch die parteiunabhängigen Oppositionsgewerkschaften.

\section{Stimmungswechsel: Das Pogrom im Juli 1983}

\subsection{Standpunkte von Regierungsvertretern}

Bei einem Uberfall tamilischer Separatisten auf einen Militärkonvoy in Tinnelveli bei Jaffna wurden am 23. 07. 198313 Soldaten getötet. Staatspräsident J. R. Jayewardene, gleichzeitig amtierender Verteidigungsminister, ordnete für den folgenden Tag ein Staatsbegräbnis in Colombo an. Aus den geplanten Trauerfeierlichkeiten entwickelte sich eine Welle von Gewalttätigkeit gegen Tamilen, die bis in die ersten Augusttage über das Land hinwegrollte. Organisierten Brandstiftungen und Morden folgten spontane Plünderungen und Angriffe gegen die tamilische Bevölkerung. Unter den Opfern befanden sich viele Tamilen indischer Herkunft. Betroffen war somit jene tamilische Bevölkerungsgruppe, die in ihrer Mehrheit separatistische Bestrebungen ablehnte.

Die Regierung hielt sich nach dem Ausbruch der Unruhen zunächst bedeckt. Erst nach vier blutigen Tagen wandte sich der Präsident in einer Fernsehrede an die Offentlichkeit. Jayewardene hatte bei früheren Konflikten ${ }^{24}$ erfolgreich seine persönliche Autorität zur Erhaltung des Friedens zwischen den Bevölkerungsgruppen eingesetzt. Damit hatte er sich Vertrauen und politische Unterstützung der Indien-Tamilen erworben. Jetzt änderte er seine Haltung. Er verstand die Ereignisse als Reaktion des singhalesischen Volkes auf den militanten Separatismus im Norden. Die Regierung wolle deshalb nun den Druck der Straße politisch nachvollziehen und sämtliche separatistischen Bestrebungen verbieten: ${ }^{25}$

"We are very sorry that this step should be taken. But I cannot see, and my government cannot see, any other way by which we can appease the natural desire and request of the Sinhala people to prevent the country being devided, and to see that those who speak for devision are not able to do so legally."

24 So z.B. während der wesentlich schwächeren Unruhen i.J. 1981.

25 J. R. Jayewardene, Fernsehrede v. 28. 7. 1983, wiedergegeben in: T.A.S.A. Dissanayaka, The Agony of Sri Lanka, (1984), S. 89. 
Die Minister G. Dissanaiake, C. Mathews und S. Thondaman, gleichfalls Präsidenten mitgliederstarker Gewerkschaften, nahmen am 05. 081983 im Parlament Stellung zu den Ereignissen. ${ }^{26}$

Der Singhalese G. Dissanaiake (Minister of Lands, Land Development and Mahaweli Development), in dessen LJEWU auch viele Tamilen und damit Opfer der Unruhen organisiert sind, sah die Ereignisse als Konsequenz des tamilischen Separatismus:

"It is true that the activities of the self-styled terrorist organization in the North combined with the short sighted and, if I might say so, unwise, policies of the representatives of the Tamil community who are in the Tamil Liberation Front created a situation in which the majority community felt terrible resentment even anger at what was going on in the North." "It is sad to think today that those who have nothing to do with the separatist cause, those who are never their sympathisers are in the refugee camps but these have been the consequences of the unleashing of Terror in the North and the incapacity of the democratic political process to persuade and to bring within the democratic fold those who were responsible for the destabilisation of the sovereignty of this country."

JSS-Präsident C. Mathews, Minister of Industries and Scientific Affairs, und Vertreter einer militant buddhistisch-singhalesischen, gegen Tamilen gerichteten Politik, blieb seinem Standpunkt treu:

"Although I am a Minister of this government, today I am speaking on behalf of the Sinhalese people of this country. During the past few days, I have met a number of people and it is their ideas I will put forward to you today. The TULF tried to trick us, they misled us. . . Either the TULF is a party that is not sincere or they have surrendered to the terrorists. Therefore they will never be able to fulfil their motives.... I am not voicing the government policy. I am speaking for the Sinhala policy. For a long period, our country enshrined Buddhism, if we do not preserve of our heritage our future generations will curse us."

"It was the tension between communities that erupted. Even today we must see why all this tension was created. We must analyse why this tension was built up. The Sinhala were frustrated for years, they were discriminated. If the Sinhala is the majority race, why can't they be the majority?"

Einzig in der Erklärung des Ministers für ’Rural Industrial Development ‘ und CWCPräsidenten S. Thondaman war Kritik an den Maßnahmen der Regierung erkennbar:

"Once again the community of Tamil speaking people of Indian origin have been made victims of a savage form of violence rendering thousands, without homes, reducing them to rags that they are now in as destitutes."

"Our grief becomes all the more pronounced in the light of the co-operation that we have extended in the past and our determination and commitment to co-exsist with the Sinhalese peacefully as brothers."

"In our thinking, it is the work of well-organised groups, who had gone on the rampage, rioting, looting and setting fire to houses and business establishments. It is more than unfortunate that these elements of disaster, these squards of goondas and rabble have been allowed to parade the streets freely, causing havoc and inflicting misery of such proportions with impunity."

26 Parlamentsreden v. 5. 8. 1983, im folgenden zit. aus: The Island, Colombo (August 1983) und Daily News, Colombo (August 1983). 


\subsection{Gewerkschaftliche Umorientierung}

Bis zu den Ausschreitungen im Juni 1983 konnten sich Präsident Jayewardene und die UNP-Regierung der Unterstützung der Indien-Tamilen sicher sein. Während des Referendums von 1982 trugen sie mit ihrer Stimmabgabe dazu bei, daß Präsident und Regierung ohne Neuwahl des Parlaments bis zumindest 1988 weiter regieren konnten. Jayewardene erzielte bei diesem Referendum eine landesweite Zustimmung von 54,7\% der gültigen Stimmen. In den Wahldistrikten Nuwara Eliya und Badulla geben die IndienTamilen mit ihrem Stimmanteil den Ausschlag. Dort erzielten Jayewardene und seine Regierung mit 73,1 \% bzw. 70,3\% die besten Ergebnisse des Landes. ${ }^{27}$

Im Gegensatz zu ihren tamilischen Brüdern im Norden sahen die Indien-Tamilen im Separatismus keine Lösung für ihre Probleme. Umgeben vom singhalesischen Siedlungsgebiet, zum Teil staatenlos, strebten sie Staatsbürgerschaft und Integration in die Gesellschaft an.

Nun war diese Bevölkerungsgruppe Hauptopfer der Ausschreitungen geworden. Die loyale Haltung gegenüber der Regierung hatte sich nicht ausgezahlt. Die Stellungnahmen des Präsidenten und der Vorsitzenden der UNP-)Brudergewerkschaften leiteten deshalb bei der CWC-Führung nach anfänglicher Verunsicherung eine Kurskorrektur ein. Der CWC setzte seine politische Zusammenarbeit mit der UNP fort, nahm aber gleichzeitig wieder Kontakte zu den Oppositionsgewerkschaften auf.

Am 15. September 1983 beriet eine Gewerkschaftskonferenz in der Parteizentrale der SLFP die Lage nach den Juli-Ereignissen. Die 19 vertretenen Gewerkschaften verfaßten folgende gemeinsame Resolution: ${ }^{28}$

Diese, in der Parteizentrale der Sri Lanka Freedom Party am 15. September 1983 abgehaltene Konferenz von Gewerkschaftsorganisationen, verurteilt entschieden die Gewalttätigkeiten, die im Lande am 24. 07. 1983 ausbrachen, und, gegen die tamilische Bevölkerung und Menschen indischer Herkunft gerichtet, für etwa 10 Tage andauerten, und drückt ihre Anteilnahme für die betroffenen Menschen aus.

Eine Reihe von Personen, die mit Arbeiterklasse und Gewerkschaftsbewegung in Verbindung stehen, wurden für mehrere Wochen inhaftiert, ohne daß Anklage gegen sie erhoben worden ist. Unter ihnen befinden sich bekannte Gewerkschafter. Organisationen, zu denen sie gehörten, wurden verboten. Wir empfinden, daß diese Situation nachteilig für die Sache von Arbeiterklasse und Gewerkschaftsbewegung ist. Diese Konferenz ruft die Regierung von Sri Lanka auf, das Verbot der Communist Party of Sri Lanka, der Nava Sama Samaja Party und der Janatha Vimukthi Peramuna aufzuheben und fordert die Freilassung aller Personen, die aus politischen Gründen in Untersuchungshaft gehalten werden.

Mehrere tausend Beschäftigte in verschiedenen Arbeitsplätzen, in großen und kleinen Betrieben, die während der kürzlichen Unruhen ernstlich beschädigt oder zerstört wurden, sind immer noch ohne Arbeit. Wir rufen die Regierung auf, Löhne und Gehälter an diese Beschäftigten zu zahlen bis sie ihre Arbeit wieder aufnehmen können oder aber passende Beschäftigung zur Verfügung zu stellen und Schadenersatz an alle Opfer der Gewalttätigkeit zu zahlen."

27 Vgl.: Recent Politics in Sri Lanka. The Presidential Election and the Referendum of 1983. W. A. Wiswa Warnapala, L. Dias Hewagama, (1983), S. $160 \mathrm{ff}$.

28 Resolution adopted at Conference of Trade Unions on 15th September 1983, unveröffentlicht, (Úbersetzung aus dem Englischen). 
Die Resolution wurde unterzeichnet von Vertretern bedeutender Oppositionsgewerkschaften, darunter der CMU, und dem CWC. Der CWC, seit 1977 gewerkschafltich und politisch eng mit der UNP verbunden, hatte begonnen, sich gewerkschaftlich von der UNP zu lösen. Die Regierung hatte diese Umorientierung durch ihre Haltung während der Juli-Unruhen selbst eingeleitet.

\section{Neue Bündnisse: Der Plantagenarbeiterstreik im April 1984}

\subsection{Ausgangslage und Ablauf}

Gegenüber den katastrophalen Lebensbedingungen der Plantagenarbeiter unter der SLFP-Regierung trat nach dem Regierungswechsel 1977 zunächst eine leichte Besserung ein. Dieser Trend kehrte sich jedoch nach 1980 wieder um. Die Arbeiter warteten auf eine Anpassung ihrer Löhne an die Preissteigerungen.

Löhne und Beschäftigungsbedingungen des staatlich-kontrollierten Plantagensektors werden hauptsächlich in 'Wages Boards' festgelegt. ${ }^{29}$ Diese mit Gewerkschaften, Arbeitgebern und Staat besetzten Ausschüsse hatten sich 1982 im Grundsatz auf eine Anpassung der Löhne geeinigt. Die Umsetzung dieser Entscheidung wurde jedoch durch Verfahrenstricks verzögert. ${ }^{30}$ So warteten die Arbeiter 1984 bei einem aktuellen Tageslohn von ca. 15.- Rs. auf eine Erhöhung um 7.- Rs., die ihnen bereits 1982 zugestanden worden war. Wegen der ohnehin schlechten Lebensbedingungen am Rande des Existenzminimums breitete sich Unmut unter den Arbeitern aus.

In dieser Situation beschloß das Joint Plantation Trade Union Committee (JPTUC), eine Arbeitsgemeinschaft oppositioneller Plantagenarbeitergewerkschaften, die überfällige Anpassung der Löhne durch gewerkschaftliche Aktionen zu forcieren. Der CWC und die UNP-kontrollierte LJEWU, jede für sich mitgliederstärker als das JPTUC, nahmen diese Diskussion auf. Zur Uberraschung des JPTUC unterstützten sie die Forderungen der Plantagenarbeiter mit einer Streikdrohung zum 1. April 1984. Die überwiegend tamilischen Plantagenarbeiter begannen sich nun von ihrer Agonie zu lösen, in die sie als Folge der Ereignisse vom Juli 1983 verfallen waren. Die Hoffnung auf eine baldige Anpassung der Löhne setzte eine gewerkschaftliche Eigendynamik in Gang. Unmittelbar vor dem 1. April vereinbarte die Regierung mit LJEWU ein Abkornmen über eine geringfügige Erhöhung der Löhne. Die Regierung hoffte, so der aufkommenden Bewegung unter den Plantagenarbeitern den Schwung zu nehmen. LJEWU wollte durch die rasche Einigung zeigen, wo die Interessen der Plantagenarbeiter inzwischen am Besten vertreten wären. Der CWC, durch die vorangegangenen Ereignisse geschwächt, sollte damit noch weiter zurückgedrängt werden. Der CWC mußte sich entscheiden. Ein Akzeptieren des ausgehandelten Ergebnisses hätte zur Folge gehabt, daß

29 Zur Funktion der Wages Boards siehe: P. Navaratne, Wages, Terms \& Conditions of Employment in Sri Lanka, (1983).

30 Plantation Strike 1984, a Ceylon Workers Congress Publication, o.J. 
der CWC als dritte Kraft zwischen Regierung (LJEWU) und Opposition (JPTUC) an Bedeutung verliert. Im Falle eines erfolgreichen Streiks hatte der CWC die Chance, als realer Machtfaktor an gewerkschaftlicher und politischer Bedeutung zu gewinnen. Ein Streik barg aber auch das Risiko, im Falle einer Niederlage die gewerkschaftliche und politische Bedeutung längerfristig oder gar endgültig zu verlieren.

Da der Zeitpunkt für einen Arbeitskampf günstig war, entschied sich der CWC für Streik. Indien hatte sich vorübergehend aus dem Teehandel zurückgezogen. Die Weltmarktpreise zogen an. In der Tee-Ernte herrschte Hochsaison. Bei einem längeren Arbeitskampf würde die Regierung auf dringend benötigte Deviseneinnahmen verzichten müssen. CWC-Präsident Thondaman hielt sich gerade in Indien auf und kehrte erst nach Streikbeginn zurück, um die Führung zu übernehmen. Damit konnte er nicht in seiner Ministerfunktion auf eine vorzeitige politische Einigung verpflichtet werden.

Der CWC war die führende Kraft dieses Streiks. CWC und JPTUC entwickelten eine gute Zusammenarbeit. Die Kontaktaufnahme mit den oppositionellen Gewerkschaften erwies sich jetzt als richtiger Schritt. Der CWC benötigte in diesem Arbeitskampf die Unterstützung anerkannter singhalesischer Führer, ${ }^{31}$ um in der ethnisch immer noch gespannten Situation eine antitamilische Stimmung zu vermeiden, die sich gegen die Plantagenarbeiter hätte richten können.

LJEWU setzte auf den Zusammenbruch der Streikfront und agierte für Wiederaufnahme der Arbeit. Mitglieder von LJEWU beteiligten sich dennoch am Streik. Der zehntägige Arbeitskampf endete mit einem ansehnlichen Erfolg für die streikenden Gewerkschaften. Die Löhne wurden mit sofortiger Wirkung weit über das von LJEWU ausgehandelte Ergebnis angehoben. Damit hatten sich Gewerkschaften erstmals in einer gröBeren Auseinandersetzung gegen die UNP-Regierung durchgesetzt.

\subsection{Neue Bündnisse}

Im Plantagenarbeiterstreik 1984 wurde deutlich, daß sich die gewerkschaftlichen Kräfteverhältnisse erneut verschoben hatten. Der CWC spielte wieder eine zentrale Rolle unter den Gewerkschaften Sri Lankas. Die politische Zusammenarbeit mit der UNP konnte den CWC nicht mehr davon abhalten, Bündnisse mit oppositionellen Gewerkschaften einzugehen. Der kommunistische "Forward" sieht eine neue Einheit der Gewerkschaftsbewegung: ${ }^{32}$

"The CWC, too, despite its governmental connection, had also attended joint meetings for some time earlier and had taken part in a joint estate strike for higher wages for plantation workers. All this showed that the basis for united trade union action was growing, and this trend should be developed without allowing sectarian consideration to take the upper hand."

31 Diese Funktion nahm u.a. der LSSP Vorsitzende Colvin R. de Silva als Vertreter der CFL Gewerkschaften wahr.

32 CPSL Zeitung Forward v. 15. 8. 1984. 
Nur die UNP-Gewerkschaften waren von dieser neuen gewerkschaftlichen Solidarität ausgeschlossen. Zu diesem Zeitpunkt hatten sie den Höhepunkt ihrer Bedeutung bereits überschritten. Ihre Kontrolle über die Arbeitnehmer Sri Lankas sank. Der CWC benötigte seine unabhängige gewerkschaftliche Kraft, um politisch als realer Machtfaktor von der UNP anerkannt zu werden. Schon aus Eigeninteresse konnte er sich eine dauerhafte Unterordnung in die UNP-Gewerkschaftsstrategie nicht leisten. Von Zeit zu Zeit mußte er beweisen, daß er diese Kraft auch einsetzen konnte.

\section{Neuere Entwicklungen}

Der CWC nutzte seine gestärkte Position, um die politischen Anliegen der Indien Tamilen voranzubringen.

Wichtigster Partner war immer noch Präsident J. R. Jayewardene, der sich inzwischen in der eigenen Partei von etlichen potentiellen Nachfolgern umgeben sah. Im Februar 1986 verabschiedete das Parlament ein Gesetz, durch das staatenlose Indien Tamilen die Staatsbürgerschaft erhalten konnten. Thondaman dankte in einer Parlamentsrede dem Präsidenten. Dabei verdeutlichte er seine spezielle Verbindung zu Jayewardene: ${ }^{33}$

"Though this relationship has been subject to much strain and stress, especially because of the anti-Tamil communal outbursts in 1977, 1981 and in 1983 in which Tamils of recent Indian origin suffered grieviously, there was never any thought of a break because of the confidence I had in him and the confidence which, I think, he has in me."

Der CWC beteiligte sich des weiteren intensiv an der Parteiendebatte um ein Dezentralisierungskonzept. Mit seinen politischen Anliegen geriet er zunehmend in Gegensatz zur SLFP. Dies wurde ein weiteres Mal deutlich, als die Regierung am 29. Juli 1987 den von der SLFP abgelehnten indo-srilankischen 'Peace Accord ' unterzeichnete. CWC und SLFP hatten in allen wesentlichen Aspekten der Tamilenfrage entgegengesetzte Auffassungen. Die SLFP-Gewerkschaften, im September 1983 noch voll Anteilnahme für die Tamilen indischer Herkunft, konnten sich dem Kurs ihrer Parteizentrale nicht entziehen.

Die Gewerkschaften des neuen Linksparteien-Bündnis SLMP/LSSP/CPSL zeigten sich in diesen Fragen eher aufgeschlossen. Dies entsprach dem Kurs ihrer Parteien, die sich von der SLFP abgrenzten.

Ein Bündnis von 22 oppositionellen Gewerkschaften nahm sich erneut gemeinsamer Anliegen an. Bei einem 'Nationalen Protesttag' am 30. Juli 1987 wurde u.a. auch die Wiedereinstellung der entlassenen Arbeiter vom 'Juli'80 Streik" gefordert. Zu einer engeren Zusammenarbeit unabhängig oppositioneller Gewerkschaften (CMU, CBEU, CPSU) mit kleineren Gewerkschaften (UPTO, CTTU) kam es zum 1. Mai 1986. 
Die innenpolitische Situation, Einschränkungen in den Aktionsmöglichkeiten und wirtschaftliche Faktoren haben die Bedingungen für die Gewerkschaftsarbeit seit 1977 erschwert. Die Gewerkschaftsbewegung Sri Lankas hat eine lange, politisch geprägte Tradition. In Einzelfragen erzielt sie hin und wieder Erfolge. Wegen ihrer andauernden Zersplitterung, die strukturell bedingt ist, war sie jedoch nicht in der Lage, die soziale Entwicklung Sri Lankas wirksam mitzugestalten. 


\title{
ABSTRACTS
}

Trade Unions in Sri Lanka: Structures and Alliances under the UNP Government

\author{
By Stefan Chrobot
}

Although extremely divided and economically not very successful, trade unions play an important role in Sri Lankan politics. After its election victory in 1977, the new UNP (United National Party) government intended to increase its control over the trade union movement. Through political cooperation with the Indian Tamil minority the integration of vast groups of plantation workers became possible. The influence and relative importance of independent and oppositional trade unions weakened during the early years of UNP government. This concept, however, later failed due to the unsolved problems of the Tamil-speaking minorities and the increasing Singhalese-Tamil conflict. The author analyses the complex structure of the divided trade union movement and the phenomenon of political sponsorship in trade unions. Based on this, the article focuses on the strike in July 1980, the anti-Tamil riots in 1983 and the successful plantation workers' strike in April 1984. The development shows changing alliances within a relatively stable structure. In the recent past, conditions for trade unions have become more difficult as a result of Sri Lanka's increasing internal conflicts.

\section{The Indo-Sri Lankan Accord in Perspective}

\section{By Lakshman M. Marasinghe}

The article traces the historical benchmarks leading to the commencement of separatist action in Sri Lanka. These historical benchmarks deal with both the pre-independence period and of the three decades of independence. The main focus of the paper is on the negotiations for a settlement. These negotiations were conducted mainly between the Sri Lankan government and the principal militant groups with the Indian government initially and subsequently playing a major role in the talks.

The article deals with seven sets of meetings, the seventh leading to the Indo-Sri Lanka Accord of July 29th, 1987. The talks initially encompassed the five main militant groups - the Tamil Eelam Liberation Organisation (TELO), the People's Liberation Organisation of Tamil Eelam (PLOTE), the Tamil Eelam Army (TEA), the Eelam Revolutionary Organisation of Students (EROS), and the Liberation Tigers of Tamil 